\title{
APLICACIÓN DE LA POS-BUROCRACIA EN LA FUNCIÓN PÚBLICA
}

\author{
APPLICATION OF POST-BUREAUCRACY IN THE CIVIL SERVICE
}

\author{
Jenny Elizabeth Parrales Reyes ${ }^{1}$, Maritza Sandra Pibaque Pionce ${ }^{2}$, Dayan Alejandra Nieto \\ Parrales $^{3}$
}

1,2,3 - Universidad Estatal del Sur de Manabí, Manabí, Ecuador

1. Email: jenny.parrales@unesum.edu.ec ORCID: https://orcid.org/0000-0001-6745-9164

2. Email: maritza.pibaque@unesum.edu.ec ORCID: https://orcid.org/0000-0002-2652-4799

3. Email: dayan.nieto@unesum.edu.ec ORCID: https://orcid.org/0000-0003-2832-1087

Recibido: 11/05/2021 Aceptado: 20/07/2021

Para Citar: Parrales Reyes, J. E., Pibaque Pionce, M. S., \& Nieto Parrales, D. A. (2021). Aplicación de la pos-burocracia en la función pública. Revista Publicando, 8(31), 36-49. https://doi.org/10.51528/rp.vol8.id2232

\begin{abstract}
Resumen:
La reforma de Estado se ha convertido en el tema central de la agenda política mundial, propuesta por la segunda generación de reformas en América Latina, provocando cambios en el sistema de organización, como generación de condiciones necesarias para la superación de los problemas más apremiantes. Por tanto, el objetivo de esta investigación es garantizar el cumplimiento de metas ante las limitaciones de la administración, mediante el acceso al servicio de la persona y sintetizar las diversas actuaciones y reformas llevadas a efecto en el sector público. El sistema político moderno, al buscar legitimación, tiene que ofrecer razones y procedimientos adecuados de generación y expresión de las decisiones. Los métodos y técnicas de investigación empleados permitieron la gestión de información y su procesamiento e interpretación para facilitar un acercamiento a esta problemática y ofrecer recomendaciones para su concreción.
\end{abstract}

Palabras clave: burocracia, cumplimiento, función pública, organización, competitividad.

\begin{abstract}
:
State reform has become the central theme of the world political agenda, proposed by the second generation of reforms in Latin America, causing changes in the organizational system, as a way of generating the necessary conditions for overcoming the most pressing problems. Therefore, the objective of this research is to guarantee the fulfillment of goals in the face of the limitations of the administration, through access to the service of the individual and to synthesize the various actions and reforms carried out in the public sector. The modern political system, in seeking legitimacy, has to offer adequate reasons and procedures for the generation and expression of decisions. The research methods and techniques employed allowed the management of information and its processing and interpretation to facilitate an approach to this problem and to offer recommendations for its realization.
\end{abstract}

Keywords: bureaucracy, compliance, public function, organization, competitiveness. 


\section{INTRODUCCIÓN}

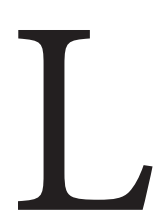

a presentación básica de las reformas realizadas apunta a mejorar la racionalidad económica, haciendo más eficiente el Estado, y pasando muchas de sus funciones a los actores más competentes, es decir de una estructuración relativamente unívoca, sostenida en una integración predominantemente vertical-escalar, de base jurídico-normativa, hacia otra progresivamente diversificada en dirección hacia una articulación 'transversal', más contingente, flexible o informal. El sistema político moderno, al buscar legitimación, tiene que ofrecer razones y procedimientos adecuados de generación y expresión de las decisiones. Las sociedades occidentales, enmarcadas en los procesos de racionalidad instrumental, "creen" en una legitimidad basada en la racionalidad y las normas, aceptan a los gobernantes elegidos de acuerdo a dichas normas y obedecen los productos de los procesos racionales y legalmente establecidos, es decir, a las leyes y no estrictamente a las personas que gobiernan.

Este trabajo investigativo se fundamenta en la búsqueda de un mejor rendimiento en las operaciones gubernamentales que han llevado a un estudio profundo de las directrices planteadas por el paradigma burocrático y ello al planteamiento de nuevos conceptos y visiones que hoy en día se conoce como paradigma posburocrático.

Para entender dicho paradigma Barzelay propone usar metafóricamente la idea de que dicho modelo es una familia ampliada de ideas: donde el prototipo burocrático es la generación anterior a la actual, y por ende se ve influenciada por su espacio y su tiempo respondiendo a las circunstancias que en su momento vivió. Los nuevos conceptos planteados guardarían estrecha relación con sus antecesores, así lo nuevo se adapta a sus circunstancias y responde a las problemáticas de la actualidad del mismo modo en que mejora las condiciones anteriormente impuestas.

Por tanto, la racionalidad quedó supeditada en diversos casos a los objetivos de obtener recursos financieros considerables en tiempos cortos. Por otra parte, el tipo de estrategias aplicadas con el personal no contribuyó a generar más racionalidad ni eficiencia. La desvalorización sistemática de la función pública, la atmósfera permanente de cortes, los criterios arbitrarios utilizados para realizarlos, la reducción del tema con frecuencia a una cuestión de ahorros presupuestarios, crearon un clima en que "activos intangibles" a los que se da crecientemente una importancia fundamental en el rendimiento, como las expectativas de carrera, el orgullo por el trabajo desempeñado, la lealtad con la organización, y la idea de servicio a la comunidad, fueron fuertemente deteriorados.

En consecuencia, la moral de trabajo se vio en una situación de incertidumbre continua, se minimizaron los incentivos no materiales. Su estrategia fue la que sustenta con precisión Evans (2003): "Los programas de austeridad que afectan significativamente las expectativas donde el servicio público es fuente de una recompensa de carrera son frecuentemente evaluados -con simplismo- en términos del ahorro en salarios que van a generar una falsa economía”.

Las contribuciones más perfeccionadas a la Nueva Gerencia Pública Internacional se 
fundamentan en concepciones culturales de un gobierno bueno y responsable. Los argumentos sobre la aceptabilidad y la eficacia de rutinas analíticas, tecnologías administrativas o estilos de supervisión. La forma básica del argumento es que seleccionar e implementar formas particulares de llevar a cabo la administración pública, puede satisfacer las aspiraciones insatisfechas del público por un gobierno bueno y responsable (Barzelay, 2003).

En el Ecuador, país dolarizado y con una grave crisis de gobernabilidad, se torna imprescindible replantear la importancia de la política fiscal y en particular de su principal instrumento el presupuesto, como elementos claves para la consolidación de la democracia y la gobernabilidad. De manera que el Estado pueda aumentar la capacidad de procesar los conflictos y presiones, no solo en el ámbito político sino también en lo económico y social, a través de prácticas democráticas y mecanismos institucionales (Mará Dolores Almeida, 2006).

\section{DESARROLLO}

En las últimas décadas en el campo de la administración pública se habla acerca del cambio, de la transformación de los gobiernos intentando mejorar su gestión, promoviendo nuevas formas de gobernar, nuevas relaciones entre los ciudadanos y sus gobiernos, así como nuevos procesos para elaborar políticas públicas. La voluntad de querer avanzar ha sido fundamental, sin embargo, ello no ha garantizado su éxito.

El nuevo paradigma ha recibido muchos nombres, además del conocido "pos burocrático" se encuentra, entre otros términos, "gerencialismo", "la nueva administración pública", "administración pública basada en el mercado" y gobierno empresarial. Según la OCDE es importante: poner más atención en los resultados y dar un mayor valor al dinero; devolver la autoridad y aumentar la flexibilidad; fortalecer la rendición de cuentas y el control; orientarse al cliente y a los servicios, robustecer la capacidad de desarrollar estrategias públicas, introducir la competencia y otros elementos del mercado, así como cambiar el tipo de relaciones que se establecen con otros niveles de gobierno (Laurence E. Lynn).

Esta disciplina que aspira a prevenir o resolver el problema de la eficacia directiva de los gobiernos, distanciándose deliberadamente del modo establecido de gobernar, y cuestionando los dos polos del eje que estructura toda actividad de gobierno: la decisión y la gestión. (Aguilar, 2007). La Nueva Gestión Pública (NGP) defiende la idea de que la eficiencia, y no la justicia, es la principal preocupación de las organizaciones públicas (Arellano y Cabrero, 2005).

El Ecuador del Siglo XXI necesita repensarse urgentemente, y dentro de este ejercicio, y quizás entre sus capítulos más importantes, está el encontrar la nueva forma de organización territorial que permita la modernización del Estado y, por ende, conseguir que la nueva gestión pública se implemente de manera inmediata en el país (Herbener, 2018).

Lo que se aspira en definitiva es buscar a través de la incorporación de principios de gobernanza y de NGP en el país, la implantación de manera permanente políticas, de las cuales los gobiernos se vean y se sientan como verdaderos estrategas, con la finalidad de brindar servicios de calidad, 
más no improvisadores y prestadores de ineficientes servicios (Herbener, 2018).

Según Barzelay (1998), es una corriente renovadora donde se agrupan teorías y modelos en su mayor parte de carácter normativo, genérico y concreto. Corrientes significativas de la NGP son: la desregulación, la agencialización, la gerencialización, la privatización, la externalización (servicios públicos gestionados por organizaciones privadas con y sin ánimo de lucro), la ética en la gestión pública, la participación ciudadana, etc., todo ello intentando vertebrar un nuevo paradigma que se ha denominado "pos burocrático".

Se hace referencia al análisis de brindar un conjunto de reflexiones sobre algunos problemas derivados de la implantación de la Nueva Gestión Pública, que es mucho más que una reforma técnica o administrativa en sentido estricto, sino que persigue la creación de una administración eficiente y eficaz, es decir que satisfaga las necesidades reales de los ciudadanos al menos costo posible e implica un esfuerzo de transformación de los marcos políticos y los juegos institucionales.

Bedón hace referencia a los cambios acontecidos en el Ecuador desde 2007 y destacó que gracias al socialismo del Buen Vivir se ha logrado encausar al país a través de una adecuada planificación, y de haber recuperado las capacidades y facultades estatales: "en este camino de construcción se creó un Estado distinto, rescatando su autoridad y ámbito de acción, colocando a la planificación participativa y descentralizada como piedra angular de las políticas públicas, dotándole de una nueva institucionalidad y de credibilidad".
Es de destacar que esta investigación promueve cuatro ejes esenciales para el país: el reto y la necesidad de innovar el Estado, transformación productiva, erradicación de la pobreza y poder popular. La gestión pública tiene como referente al Plan del buen vivir.

La nueva gestión pública persigue la creación de una administración eficiente y eficaz es decir una administración que satisfaga las necesidades reales de los ciudadanos al menor coste posible, favoreciendo para ello la introducción de mecanismos de competencia que permitan la elección de los usuarios y a su vez promuevan el desarrollo de servicios de mayor calidad, bajo sistemas de control que otorguen plena transparencia de los procesos planes y resultados, para que por un lado perfeccionen el sistema de elección, y por otro favorezcan la participación ciudadana (Sánchez, 2007).

La administración pública es una disciplina que aspira prevenir o resolver el problema de la eficacia directiva de los gobiernos, distanciándose deliberadamente del modo establecido de gobernar, y cuestionando los dos polos del eje que estructura toda actividad de gobierno: la decisión y la gestión (Aguilar, 2007).

La NGP defiende la idea de que la eficiencia, y no la justicia, es la principal preocupación de las organizaciones públicas (Arellano y Cabrero, 2005). Se distingue por organizaciones orientadas a resultados más que a procedimientos (por procesos más que por funciones), y hacia los ciudadanos-clientes más que hacia los superiores inmediatos, el costo eficiencia de las operaciones, la presupuestación por resultados, el establecimiento de agencias ejecutivas autónomas 
para fines públicos específicos, la contratación de organizaciones privadas y sociales para la prestación de determinados servicios públicos, el establecimiento de contratos particulares entre el superior y los funcionarios (entre el principal y los agentes) en sustitución de normas generales, los sistemas de medición y evaluación del desempeño, el gobierno digital, etc.

La finalidad de la NGP está orientada a dar forma a una estructura administrativa descentralizada y desregulada, que fomente la iniciativa del personal y su compromiso con el logro de resultados, para lo cual se otorga al personal la capacidad de decisión y se le responsabiliza por el resultado. Su tesis central es que la descentralización y la eficiencia son interdependientes, en sentido contrario a la idea de que la centralización jerárquica y la reglamentación precisa son las condiciones de la eficiencia (Arellano y Cabrero, 2005).

La NGP, ha prestado atención a la administración pública, ha cuestionado su estructura burocrática de organización y operación -la centralización jerárquica y la reglamentación precisa e inalterable- y se ha presentado como una disciplina o práctica profesional que posee los métodos de reorganización y de trabajo, apropiados para incrementar la eficiencia y mejorar la calidad de la gestión gubernamental.

En términos organizacionales la NGP defiende que las organizaciones públicas tienen que ser controladas. No corresponde a los burócratas (o gerentes públicos como le gusta llamarlos a la Nueva Gestión Pública) definir qué es bueno o malo para la sociedad, son los individuos (como ciudadanos o, mejor aún, clientes) quienes mejor conocen lo que quieren. Pero queda claro que incluso los ciudadanos necesitan ayuda para poder definir los que es mejor para ellos. De modo que es posible decir que la NGP apoya la participación de los ciudadanos una vez que el diseño del programa público ha pasado por los mecanismos de mercado (Ostrom, 1973).

Es preciso referenciar los principales problemas que ha enfrentado la NGP según Aguilar (2007) están relacionados con:

\section{- La definición del modelo de Estado.}

Se debe definir el modelo de Estado y después buscar los mejores instrumentos de gestión acorde con los objetivos y condicionantes del modelo seleccionado, Es decir, el cómo (forma de gestionar) acaba definiendo el qué (modelo de Estado). En este sentido hay que abandonar la idea que la NGP es un conjunto de instrumentos para la mejora de la gestión y hay que considerar la incorporación de valores ideológicos y administrativos.

En una esfera algo más progresista algunos instrumentos de la NGP pueden asociarse a las formulaciones británicas de la "tercera vía" de Blair (1998) y Giddens (1999) en lo que de forma más correcta se puede denominar como sociedad del bienestar. De la misma forma, los instrumentos de la NGP priorizan más la eficiencia, tanto del mercado como del sector público, por encima de otros criterios como puede ser la equidad.

- El isomorfismo institucional: los problemas de imitar modelos institucionales de otras tradiciones administrativas. 
Estos procesos hacen referencia a la tendencia de ciertas organizaciones a adoptar fórmulas institucionales aceptadas y consolidadas en su entorno de referencia. Esta reproducción puede darse a través de dos tipos de isomorfismo institucional (DiMaggio \& Powell, 1991): el Isomorfismo coercitivo y el Isomorfismo mimético.

Los procesos de institucionalización se refieren a las dinámicas a través de las cuales una determinada estructura, creencia o actividad se inserta en el marco institucional, es decir, se acepta sin discusión y se incluye en los mecanismos de reproducción del isomorfismo institucional. En este proceso de institucionalización, suelen aparecer situaciones de decoupling, entendidas como desajustes entre las instituciones formales y el funcionamiento efectivo de la organización.

El decoupling es una respuesta de la organización al isomorfismo institucional cuando éste entra en conflicto con sus requisitos mínimos de eficacia y eficiencia, lo que requiere cierta flexibilidad en la interpretación de las instituciones formales para que puedan ser aceptadas por los distintos agentes implicados. El fenómeno del decoupling y la incapacidad de copiar los "aspectos ocultos y más finos" de las estrategias de modernización de la NGP son dos razones que pueden explicar el fracaso de la implantación de los parámetros de ésta en los países latinos.

- $\quad$ El impacto sobre la cultura organizativa: las implicaciones de una cultura empresarial en el sector público.
La esencia del enfoque de cultura organizativa reside en considerar a las organizaciones como sociedades relativamente autónomas dotadas de sus propios procesos de socialización, de normas y estructuras sociales.

Impulsar la cultura neo-empresarial genera una cierta convulsión inicial que se supera con la convicción de los conversos ya que los valores previos escasamente articulados generaban incertidumbre entre los miembros de la organización pública y, en cambio, la nueva cultura otorga un sentido a todo, incluso a lo escasamente explicable o justificable desde un punto de vista de la ética pública. En esta situación de debilidad cultural de lo público, la introducción de valores neoempresariales de la mano de la NGP es muy negativa ya que los medios (economía, eficacia $\mathrm{y}$ eficiencia) se transforman en los fines y se pierden de vista los objetivos últimos de los programas públicos y a los colectivos de ciudadanos a los que van dirigidos.

Pero el impacto de las corrientes neoempresariales de la NGP no se limita a la transformación de la cultura y de los valores de los empleados públicos y tiene también su impacto en otras colectivos como los cargos políticos la ciudadanía: Los cargos electos y de confianza política se han encargado de modernizar unas administraciones públicas muy anticuadas, Los ciudadanos, tradicionalmente despreocupados por la Administración pública, adoptan una posición que mezcla la desconfianza con la crítica fácil.

- Las dificultades de la Administración Relacional en un contexto de gobierno 
multinivel: ¿quién controla la agenda? y los problemas derivados de las "capturas".

La Administración Relacional responde a un modelo en que el aparato administrativo tradicional reduce de forma drástica sus dimensiones reservándose la capacidad de planificación, decisión, control y evaluación y difiriendo la gestión directa de los servicios públicos a una red de instituciones públicas $y$, fundamentalmente, privadas. Además de gestionar siguiendo un modelo de Administración Relacional ello se hace en un contexto de gobierno multinivel que se deriva de la multiplicidad de niveles administrativos y de administraciones que posee cada país. El modelo de la administración relacional se fundamenta en la externalización de los servicios públicos y los valores neoempresariales. La combinación de la externalización con la cultura neoempresarial, que implica un notable déficit de valores públicos en los aparatos administrativos.

Las estrategias de modernización de la NGP tienen como principal logro diseñar unos sistemas administrativos flexibles. $\mathrm{La}$ flexibilidad se considera, desde hace años, como una virtud de los sistemas administrativos más modernos que han influido como postburocráticos (Barzelay, 1998).

Los principales postulados de la teoría para la mejora de las políticas y la administración están enfocados en las operaciones del gobierno y su aplicabilidad se basan en dos principales tributarios de esta corriente.
La gestión de procesos comerciales y la gestión del rendimiento son, sin lugar a dudas, pertinentes para la discusión de la administración de las operaciones del gobierno. Sin embargo, se requiere cierta ingenuidad para hacer corresponder esas escuelas de pensamiento con las circunstancias del sector público. Por lo general, se toma como condición de fondo que el diseño organizacional se parece más a una estructura jerárquica sencilla que a una burocracia profesional. La vitalidad de esta corriente depende de conocer bastante los desarrollos relativamente nuevos en disciplinas funcionales como a contabilidad y control administrativo (Barcelay, 2003).

Según Morande (2016):

- La aplicación de las medidas económicas de corte neoliberal, planteadas en el Consenso de Washington, marcaron el punto de partida de una nueva crisis política y social en América Latina.

- Estas medidas, conocidas como el Consenso de Washington, marcaron el inicio de una serie de reformas políticas y económicas en los países latinoamericanos, y generaron un impacto social de carácter pernicioso.

- El Consenso de Washington ha sido considerado uno de los principales obstáculos al desarrollo de los países en Latinoamérica a partir de la década de los noventa. Este tema presenta relevancia a nivel social debido a que la 4 imposición de medidas neoliberales se vio reflejada en dichas políticas además de la ineficiencia de muchos gobernantes en la región.

La estructura planteada por la Reforma, la 
nueva administración estipula cuatro tipos de control:

- Control de resultados, realizado a partir de indicadores de desempeño estipulados de forma precisa en los contratos de gestión.

- Control contable de costos, que abarcará no sólo el control de los gastos realizados, sino también el descubrimiento de formas más económicas y eficientes de hacer cumplir las políticas públicas. A partir de esto, los funcionarios públicos comenzaron a valorar el buen uso del dinero público, y así a respetar más a la población pensada como contribuyente (Metcalfe \& Richards, 1989).

- Control por competencia administrada, o por cuasi-mercados, en los cuales las diversas agencias buscan ofrecer el mejor servicio a los usuarios. Esta competencia puede traer ganancias de eficiencia y efectividad al sistema, ya que la pugna obliga a una utilización más racional de los recursos y porque la tendencia es a aumentar la gama de servicios a disposición de los ciudadanos.

- Control social, por medio del cual los ciudadanos evaluarán los servicios públicos o participarán en la gestión de los mismos. El control social es un instrumento fundamental para manejar la compleja relación entre los ciudadanos y sus agentes, los políticos, y los burócratas. Se ha mostrado que el control de la población sobre la burocracia - supervisión de tipo "alarma de incendio" - es lo que asegura la mayor optimización de la relación entre el principal (ciudadanos) y sus agentes gubernamentales, toda vez que el costo de la información en este caso es menor que el que corresponde a la relación establecida entre políticos y burócratas, y que las ganancias inmediatas para la sociedad son mayores (McCubbins \& Schwartz, 1984).

Los cambios sociales, económicos y políticos ocurridos en América Latina en los últimos 25 años del siglo XX no trajeron mayores alteraciones en las relaciones entre Estado, mercado y sociedad propias del modelo desarrollista que imperó en la región durante el período de 1930 a 1970. Se ha perpetuado el dominio del Estado con sus prácticas autoritarias, clientelistas e individualistas que favorecen los intereses de algunos grupos, como las clases altas o las elites políticas, en detrimento del bienestar de los diversos sectores y asociaciones que conforman la sociedad civil.

Estos cambios desde el ámbito internacional, especialmente en España, Grecia y Portugal, y la crisis de la deuda de algunos países con la banca internacional, como México, provocaron demandas de mayor atención y participación de la sociedad en asuntos públicos y políticos; situación que fue el inicio, a principios de los 80, de un período de transición a la democracia, que aún sigue en proceso. En un inicio la llegada de este nuevo orden político fue promisoria; colapsaron las dictaduras militares y políticas, el Estado se acercó a las necesidades y problemas de la sociedad, pero con el tiempo esta tendencia se fue desluciendo.

Como afirma Weffort (1995, p.411), la modernidad en América Latina ha tenido un rumbo equivocado que, en lugar de promover un orden armónico y consensual, ha creado las condiciones para que se establezca una "anomia generalizada"; modernidad que, en vez de permitir 
la emergencia de una sociedad civil unida e integrada sobre la base de marcos culturales y conceptuales determinados, la ha fragmentado al estilo de los tiempos medievales. Por ejemplo, en Chile, como lo argumenta De la Maza (2002, p.237), la transición a la democracia permitió una mayor inclusión, pero se mantuvieron las diferencias sociales, la débil integración interna de los grupos y el Estado incluyente y elitista que generó, lo que el PNUD denominó "el malestar ciudadano".

La NGP apareció en el medio latinoamericano en los años 80 y 90 como propuestas disciplinarias y profesionales para superar la vulnerabilidad financiera o la crisis fiscal en que habían caído los Estados sociales desarrolladores y/o para restablecer la naturaleza pública que las políticas de los gobiernos autoritarios habían tergiversado o pervertido y/o para mejorar los servicios públicos que eran considerados insatisfactorios en calidad, cobertura y costo eficiencia.

Se infiere entonces que el principio de la "nueva riqueza de las naciones" se ve hoy en función principalmente de la calidad de las instituciones y de sus capacidades de acción colectiva (Prats, 2000).

De acuerdo con Moore, las técnicas de dirección pública más importantes son la estrategia organizativa y la gestión política.

La estrategia organizativa debe satisfacer tres pruebas:

- Ser valiosa para los agentes críticos a un coste bajo en dinero y autoridad.

- Ser legítima y políticamente sostenible; es decir, atraer sostenidamente autoridad y dinero.

- Ser operativa y administrativamente viable.

La gestión política consiste en: construir un clima de tolerancia, apoyo activo o asistencia operativa, en concordancia con las técnicas de la gestión política: la promoción empresarial, la gestión del desarrollo de la política, la negociación, la deliberación pública, el aprendizaje social y el liderazgo, el marketing público y la comunicación estratégica

\section{a. La promoción empresarial}

- Implica conocer quién estará implicado o afectado por la política o estrategia correspondiente.

- Hay que valorar y conocer la postura de los diferentes actores implicados, lo cual exige intuir y si es posible investigar sus actitudes frente a la estrategia.

- Hay que definir el grado de publicidad de las acciones de acuerdo con la materia de que se trate.

- $\quad$ Es preciso formular los temas de manera que maximicen apoyos y minimicen resistencias.

- Hay que actuar en el momento favorable. Los temas entran en la agenda no por su importancia, sino por la posibilidad de actuación en el tema.

- No olvidar que el trabajo se hace a medio plazo, lo cual permite ir cambiando el escenario a favor de la estrategia. Disponer de una buena red de contactos, de conocimientos y credibilidad son clave para el éxito. 


\section{b. La gestión del desarrollo de la política}

En la promoción empresarial se prioriza dos virtudes clave para el éxito:

- Virtudes procedimentales: la política emerge de un amplio proceso de consulta, todas las partes interesadas han debido ser oídas.

- Virtudes sustantivas: los hechos relevantes están disponibles, dispuestos en un marco en el que se presentan los valores en juego, las principales alternativas que los decisores pueden escoger y las consecuencias previsibles de las mismas, pero, además, es preciso tomar decisiones dentro de la organización, y para ello hay que:

- $\quad$ Identificar el tema

- Valorar la importancia y urgencia del tema

- $\quad$ Analizar políticamente el tema

- $\quad$ Analizar sustantivamente el tema

- Garantizar un proceso justo a los stakeholders o actores afectados por el tema

- Relacionar los temas entre sí y con la estrategia global

- $\quad$ Seguir el tema

- $\quad$ Supervisar y evaluar su puesta en práctica.

\section{c. Negociación}

- La pregunta inicial es: ¿existe tema para negociar?
- $\quad$ Si es así, identificar los intereses de los actores

- La mejor alternativa al acuerdo negociado es el punto que marca si seguir negociando merece la pena. Si la negociación no mejora el beneficio obtenido sin negociar, no se siente a la mesa.

- Otra pregunta clave: ¿la negociación aporta valor a ambas partes?

- Conviene añadir temas y actores para crear valor

- Un buen directivo es capaz de influir en las alternativas a la negociación para lograr su meta. d. Deliberación pública, aprendizaje y liderazgo social.

- Es importante fomentar la deliberación pública, para ello es necesario creer en la razonabilidad de los actores. Ello no obsta a que haya que guiar el proceso.

- También hay que fomentar la movilización de los ciudadanos para que puedan actuar por su cuenta.

- $\quad$ Es importante ofrecer consejos durante el proceso. -También es necesario guiar el aprendizaje.

- $\quad$ Y hay que adecuar el ritmo a la tensión del proceso. e. Marketing público y comunicación estratégica

- Implica que hay que impulsar políticas haciéndolas comprensibles, consiguiendo el apoyo y la cooperación de los afectados.

- Informar no es hacer propaganda. Hay que informar, no manipular. 
- $\quad$ Es imprescindible determinar los atributos de la actividad gubernamental que los que pagan por el producto consideran relevantes.

- Mantener el buen funcionamiento de la organización: ajustar estructuras, gestionar RR.HH., formar y desarrollar, etc., movilizar a los coproductores, averiguar el tipo y grado de innovación necesarios, trabajar en equipo.

\section{MATERIALES Y MÉTODOS}

Este trabajo investigativo aplicó la metodología de revisión bibliográfica, mediante una macro búsqueda que permitió la identificación de los documentos referentes al tema. Las estrategias de búsqueda, organización y análisis de la información, proporcionaron tanto la obtención de los documentos referentes al tema de investigación, así como su sistematización y estructuración con el objetivo de garantizar el cumplimiento de metas ante las limitaciones de la administración, mediante el acceso al servicio de la persona y sintetizar las diversas actuaciones y reformas llevadas a efecto en el sector público.

\section{RESULTADOS}

Esta investigación sustenta sus resultados de la nueva gestión pública en concordancia con el acceso al servicio de la persona y las diversas actuaciones y reformas llevadas a efecto en el sector público, caracterizada por:

1. La preocupación por la eficiencia ocupa un lugar preeminente. Los programas de privatización en todo el ámbito de los monopolios naturales se combinan con fuerte subcontratación en áreas de infraestructuras e, incluso, sociales. La llegada de nuevos actores impulsa la posible introducción de competencia entre el sector público y el privado por la gestión de servicios (es el market testing británico), o la competencia entre organismos públicos, previamente estructurados como fundaciones o agencias, por un mismo presupuesto o unos mismos clientes.

2. Mejora de la capacidad técnica de seguimiento del rendimiento de la Administración. A través de auditorías contables, financieras y económicas; de la evaluación de programas que se incorporan como requisito para la renovación del propio programa; mediante las tecnologías de la información aplicadas al control contable; mejorando los procesos de elaboración y seguimiento presupuestario, etc.

3. Desarrollo de criterios gerenciales con el énfasis en aspectos como la gestión de los rendimientos (numerosas agencias trabajan con contrato programa o se exige la elaboración de planes estratégicos a controlar anualmente, como en la estadounidense Government Performance and Results Act"), la fragmentación de grandes unidades en centros de responsabilidad por tipo de usuarios, y la introducción del concepto de cliente en la configuración del servicio.

4. Deterioro de la estructura estatutaria tradicional de la Administración pública. Frente a la uniformidad salarial y el ascenso por antigüedad se implanta la evaluación del rendimiento individual o por grupo; frente a la permanencia en el empleo se introducen mecanismos de reducción plantillas y de eliminación de excedentes de personal; frente a la determinación unilateral de condiciones de trabajo se acepta la negociación colectiva con limitaciones.

5.Democratización relativa de la gestión y preocupación por la receptividad. Hacia el 
exterior, mediante las encuestas al usuario, la simplificación de procedimientos, la mejora de los puntos de contacto, la clarificación del lenguaje administrativo, la formación de los informadores y del personal de "ventanilla", la apertura de información y de consulta de archivos, los núcleos de intervención operativa, las conferencias de consenso, los grupos de mediación; hacia el interior, mejorando los servicios de asesoramiento del empleado, preocupándose por la prevención de riesgos laborales y la ergonomía, introduciendo mecanismos de empowerment y participación, como los círculos de calidad, etc.

6.Reorganización de las estructuras. Mediante sistemas organizativos más flexibles, reducción de niveles intermedios, creación de agencias independientes, promoviendo la descentralización territorial $y$ funcional $y$, sobre todo, desarrollándose el trabajo en red.

7. Transformación de la cultura interna. Mediante procesos de formación continua y de desarrollo organizativo se intenta introducir una cultura de servicio al cliente, frente a la cultura legalista y de división competencial estricta.

8. Generación de nuevos instrumentos de lucha contra la corrupción y de construcción de infraestructura ética.

La llegada a este estado ha sido progresiva. Si se analiza el sistema de valores que ha enmarcado la acción de la Administración en estos 30 últimos años, se puede constatar que se ha pasado de una Administración esencialmente, aunque no totalmente, preocupada por la imparcialidad y la aplicación objetiva de la ley en el marco del paradigma burocrático, a una Administración preocupada ante todo por la eficiencia y la economía, especialmente en los países anglosajones y durante los primeros años de la reforma conservadora de los 1980s; posteriormente, en los 1990s, a una Administración que retoma la eficacia y, sobre todo, que se abre a la innovación y flexibilidad, para acabar más recientemente con la priorización de valores vinculados a la democracia, como la transparencia, la participación o la contabilidad.

\section{CONCLUSIONES}

Es de indicar que la generación de ideas más joven ha evolucionado a partir del paradigma burocrático. El propósito de las reformas burocráticas era permitir al gobierno ponerse al servicio del interés público. El gobierno serviría al interés público si era honrado y eficiente. Con todo esto se logró delinear la idea de interés público, ya que el principal fin era servir los intereses de la población. La premisa se cimentaba en el reclutamiento de expertos en las áreas de contabilidad, ingeniería y trabajo social.

Los expertos basaban su trabajo en la experiencia y habilidad en sus campos de estudio, con ello se orientaba mediante sus premisas básicas de lo que para ellos era la solución a determinado problema, enfrentándose después a la evaluación ciudadana, quien constantemente estaba insatisfecha ante su trabajo. Por su parte el paradigma posburocrático propone sustituir la idea de interés público por los resultados que valoran los ciudadanos, sintetizar las diversas actuaciones y reformas llevadas a efecto en el sector público, porque cuando se habla de Nueva Gestión Pública en sentido estricto se refiere a la Administración centrada en valores de eficiencia y eficacia, mediante el acceso al servicio de la 


\section{R E V I S T A}

\section{Public a n d o}

I S S N $13990-9304$

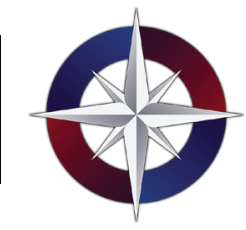

persona y sintetizar las diversas actuaciones y reformas llevadas a efecto en el sector público. 


\section{REFERENCIA}

Aguilar, L. (2007). El aporte de la Política Pública y de la Nueva Gestión Pública a la Gobernanza.

Arrellano y Cabrero (2005). La Nueva Gestión Pública y su teoría de la organización.

Barzelay, M. (1998): Atravesando la Burocracia. Una Nueva Perspectiva de la Administración Pública, México, Fondo de Cultura Económica.

Barzelay, (2003). La Nueva Gerencia Pública Invitación a un Dialogo Cosmopolita. II Semestre, Vol 12, Centro de Investigación y Docencia Económicas (CIDE) pp241-252.

Blair, T. (1998): La Tercera Vía por Tony Blair, Madrid, El País Aguilar.

DiMaggio, P. \& Powell, W. (1991): The New Institutionalism in Organizational Analysis, Chicago, Chicago University Press.

De la Maza, G. (2002). Sociedad Civil y democracia. México. Fondo de Cultura Económica: Sociedad civil, esfera pública y democratización en América Latina: Andes y cono sur, p.211-240.

Giddens, A. (1999): La Tercera Vía. La Renovación de la Socialdemocracia, Madrid, Taurus.

McCubbins, M. \& Schwartz, T. (1984). "Congressional Oversight Overlooked: Police Patrols versus Fire Alarms", American Journal of Political Science, n.28.

Metcalfe, L. \& Richards, S. (1989). La Modernización de la Gestión Pública, INAP, Madrid, página 50.
Morande, L. (2016). A casi tres décadas del Consenso de Washington ¿Cuál es su legado en América Latina? Revista Estudios Internacionales 185. Instituto de Estudios Internacionales - Universidad de Chile, ISSN 0716-0240, Págs. 31-58.

Ostrom, E. (1973). Una perspectiva del capital social desde las ciencias sociales: capital social y acción colectiva. Revista Mexicana de Sociología 65 (1): 155-233.

Prats, J. (2000): “Reforma del Estado y Desarrollo Humano: una Correlación Problemática”, Quórum, núm. 1.

Ramio, Matas, C. (2001). Los problemas de la implantación de la Nueva Gestión Pública en las Administraciones Públicas Latinas.

Sánchez, G.(2007). La nueva gestión pública: Evolución y tendencias. Universidad de Salamanca.

Weffort, F. (1995). La América Latina equivocada: apuntes sobre democracia y la modernidad en la crisis de América Latina. México. Fondo de Cultura Económica: América Latina a fines de siglo, p. 399-432. 